\title{
Silver Nanoparticles Decorated on a Three-Dimensional Graphene Scaffold for Electrochemical Applications
}

\author{
A. Bello ${ }^{a}$, M. Fabiane ${ }^{a}$, D. Dodoo-Arhin ${ }^{a}$, K.I. Ozoemena ${ }^{\text {b,c }}$ and N. Manyala ${ }^{a^{*}}$ \\ ${ }^{a}$ Department of Physics, Institute of Applied Materials, SARChI Chair in Carbon Technology and Materials, \\ University of Pretoria, Pretoria 0002, South Africa \\ ${ }^{\mathrm{b}}$ Council for Scientific and Industrial Research, Meiring Naude Road, Brummeria, PO Box 395, Pretoria 0001, \\ South Africa \\ ${ }^{c}$ Department of Chemistry, University of Pretoria, Pretoria 0002, South Africa \\ *Email: ncholu.manyala@up.ac.za
}

\begin{abstract}
Silver metal nanoparticles were decorated by electron beam evaporation on graphene foam (GF) grown by chemical vapour deposition. X-ray diffraction, Raman spectroscopy, scanning and transmission electron microscopy, and atomic force microscopy were used to investigate the structure and morphology of the graphene foam/silver nanoparticles (GF/Ag). Both samples were tested as electrodes for supercapacitors. The GF/Ag exhibited a significantly higher capacitive performance, including a specific capacitance value of $\left(\sim 110 \mathrm{Fg}^{-1}\right)$ and excellent cyclability in a three-electrode electrochemical cell. These results demonstrate that graphene foam could be an excellent platform for metal particles for investigating improved electrochemical performance.
\end{abstract}

Keywords: Graphene foam; CVD; electron beam evaporation; Silver nanoparticles; electrochemical capacitor. 


\section{Introduction}

The discovery of graphene has inspired much research activity to understand its unique physicochemical properties and possible applications. Graphene [1] is a single layer of graphite consisting of a two-dimensional honeycomb lattice of $\mathrm{sp}^{2}$-bonded carbon atoms with a unique electronic structure that exhibits linear dispersion at a high symmetry point in the reciprocal space, resulting in effective dynamics of electrons [2]. It has been touted as an electrode material due to its theoretically high surface area $\left(2630 \mathrm{~m}^{2} \mathrm{~g}^{-1}\right)$, high electrical conductivity, chemical stability and excellent mechanical properties [3-9]. Graphene is thus being considered as a building block for electrochemically active materials [10] and as a suitable platform for the loading of metal nanoparticles [11]. These subtle properties make graphene exceptional for both micro- and macroscopic applications.

Recently, graphene-metal composites have been considered as electrode materials for electrochemical applications [12]. Metal nanoparticles such as $\mathrm{Ag}, \mathrm{Pd}, \mathrm{Pt}$ and $\mathrm{Au}$ exhibit large surface area to volume ratios at the nanoscale and hence are excellent materials where large surface areas are required. A variety of successful approaches for preparing graphenemetal composites have been reported. For instance, electrodeposition [13,14], chemical reduction [15-18], epitaxial deposition [19] and chemical vapour deposition [20] have been used. However, it is usually difficult to achieve uniform and homogeneous coating of the graphene surface through some of these deposition techniques [21].

Chen et al. [22] reported on three-dimensional (3D) flexible and conductive interconnected graphene networks grown by chemical vapour deposition (CVD) using a nickel foam template. Since then, the 3D form of graphene has attracted application in fuel cells [23], batteries [24] and electrochemical cells [25] due to its high porosity, high specific surface area and high electrical conductivity. 
To the best of our knowledge, only a few pioneering studies on 3D graphene-metal composites have been done [23, 25-28]. In this study, we report on the enhanced electrochemical performance of CVD-grown graphene foam (GF) decorated with silver nanoparticles using a physical technique (electron beam evaporation). The electrochemical effect of the Ag nanoparticles on the GF is discussed. We chose silver nanoparticles (as model electro-active nanoparticles) due to the fact that they possess a very high surface area to volume ratio, and have been shown to exhibit electrochemical properties that make them suitable for potential application in electrochemical devices such as sensors [29-31] and in fuel cells (i.e. hydrogen-evolution reaction [32] and oxygen-reduction reaction [33]).

\section{Experimental}

Graphene foam (GF) was synthesised by CVD onto a catalytic nickel foam (Alantum Innovation in alloy foam, Munich, Germany), $420 \mathrm{gm}^{-2}$ in areal density and $1.6 \mathrm{~mm}$ in thickness. The details of the synthesis procedure are given in our previous paper [34]. To provide mechanical support for the 3D structure of the GF during etching of the nickel, polymethylmethacrylate (PMMA) was drop-coated onto the sample and baked at $180{ }^{\circ} \mathrm{C}$ for 30 minutes. This solidifies the PMMA onto the nickel-graphene surface. The samples were placed in a $3 \mathrm{M} \mathrm{HCl}$ solution at $80{ }^{\circ} \mathrm{C}$ and left overnight to ensure that the nickel was completely etched away. The GF was then placed in acetone at $50{ }^{\circ} \mathrm{C}$ to remove the PMMA, after which the samples were rinsed in deionised water and then dried. Ag particles were deposited on the GF at a rate of $0.5 \AA / \mathrm{min}$ in a $10^{-7}$ mbar vacuum using a standard electron beam evaporator with an e-Vap CVS-10 (MDC) power supply capable of accelerating electrons at $10 \mathrm{keV}$ energy and equipped with a crystal monitor for film-thickness monitoring. 
Raman spectroscopy data were collected on a Jobin Yvon Horiba TX 6400 micro-Raman spectrometer equipped with a triple monochromator system to eliminate contributions from Rayleigh lines and using LabSpec (Ver. 5.78.24) analytical software. All the samples were analysed with a $514 \mathrm{~nm}$ argon excitation laser $(1.5 \mathrm{~mW}$ laser power on the sample to avoid thermal effects), a 50x objective with recording times ranging from $120 \mathrm{~s}$ and a resolution of $2 \mathrm{~cm}^{-1}$. To confirm the presence of the Ag nanoparticles and the corresponding microstructure, X-ray powder diffraction (XRD) patterns of the GF samples were collected on an XPERT-PRO diffractometer (PANalytical BV, Netherlands) with theta/theta geometry, operating a cobalt tube at $35 \mathrm{kV}$ and $50 \mathrm{~mA}$. The goniometer is equipped with an automatic divergence slit and a PW3064 spinner stage. The instrumental resolution function was characterized with the NIST SRM 660a (LaB6) standard. The XRD patterns of all samples were recorded in the $40.0^{\circ}-80^{\circ} 2 \theta$ range with a step size of $0.017^{\circ}$ and a counting time of 15.240 s per step. Qualitative phase analysis of the samples was conducted using the X'pert Highscore search-match software.

Morphological characterization of the samples was performed on an Agilent 5500 atomic force microscope in acoustic mode at a frequency of $\sim 150 \mathrm{kHz}$. Images were obtained with a high-resolution Zeiss Ultra Plus 55 field emission scanning electron microscope (FE-SEM) operated at $2.0 \mathrm{kV}$, and with a JEOL JEM-2100F transmission electron microscope (TEM) operated at $200 \mathrm{kV}$.

Finally, electrochemical measurements such as cyclic voltammetry (CV) and galvanostatic charge-discharge were performed using an Autolab PGSTAT workstation 302 (ECOCHEMIE) driven by GPES software in a three-electrode setup. The GF/Ag served as the working electrode, a glassy carbon plate as the counter electrode, $\mathrm{Ag} / \mathrm{AgCl}(3 \mathrm{M} \mathrm{KCl})$ as the 
reference electrode and $2 \mathrm{M} \mathrm{KOH}$ was used as the electrolyte. Electrochemical impedance spectroscopy (EIS) was performed in the frequency range of $100 \mathrm{kHz}-10 \mathrm{mHz}$.

The electrical resistance of both samples was measured via the four-point method. The current and voltage were provided by a source-measure unit (Keithley 2636B). The values of applied current, I, and their corresponding voltages, V, were recorded and the resistance, R, was calculated from Ohm's law, i.e. from $\mathrm{R}=\mathrm{V} / \mathrm{I}$. The cross-sectional area of the samples was calculated and the conductivity, $\sigma$, was then estimated according to $\sigma=1 / \mathrm{AR}$.
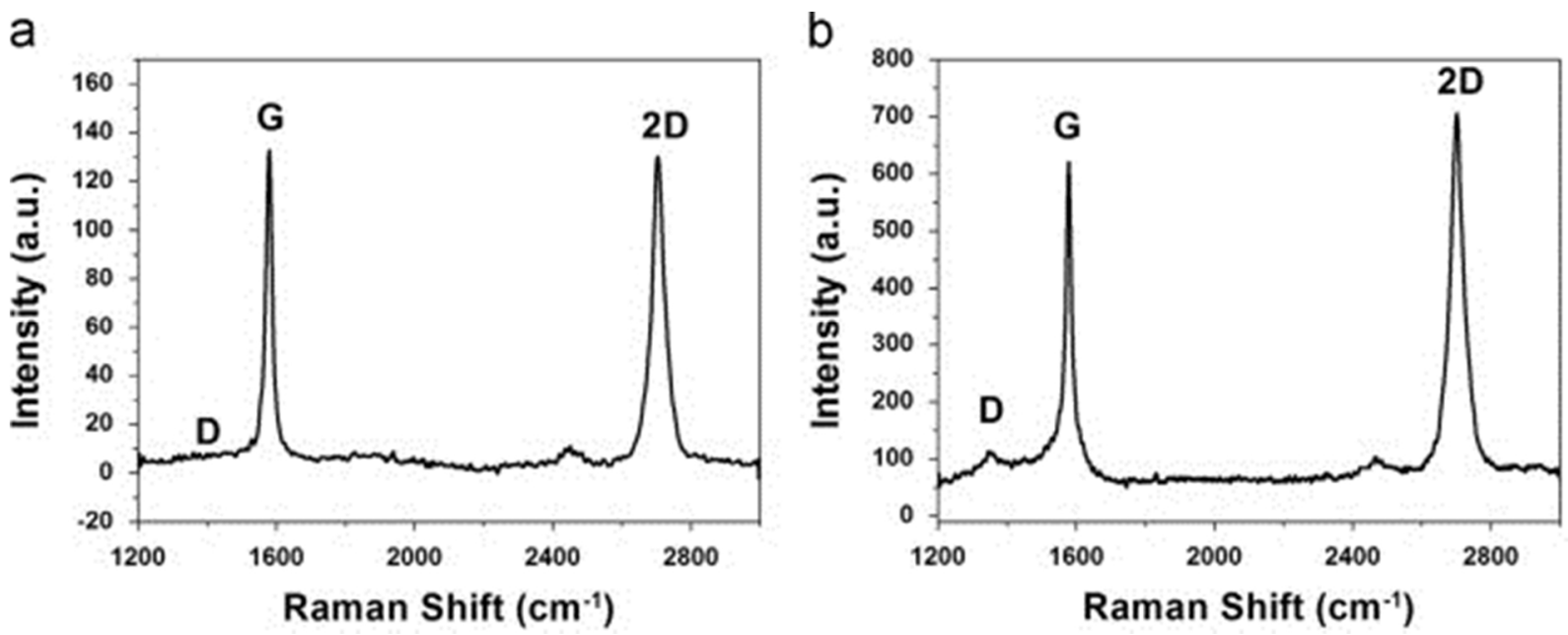

Figure 1. Raman spectra of the samples: (a) graphene foam (GF); (b) graphene foam with silver nanoparticles $(\mathrm{GF} / \mathrm{Ag})$

\section{Results and discussion}

Figure 1 (a) shows the Raman spectrum of the GF which consisted of two major peaks at $\left(1578 \mathrm{~cm}^{-1}\right)$ and $\left(2703 \mathrm{~cm}^{-1}\right)$, corresponding to the typical G- and 2D-bands of graphene [35]. The absence of the D-peak (disorder) around $1350 \mathrm{~cm}^{-1}$ on the spectrum shows that the GF is of good quality [36]. Figure 1 (b) shows the Raman spectrum of the GF/Ag sample. The appearance of a D-peak at $1350 \mathrm{~cm}^{-1}$ could be attributed to the strain effect on the atomic position of the carbon atoms caused by the Ag particles. Both Raman spectra show a weak bands at $\mathrm{G}^{*}\left(2450 \mathrm{~cm}^{-1}\right)$ which originates from the double resonance process involving two 
iTO phonons around the K-point in the phonon dispersion of a two-dimensional graphite [37]. The $2 \mathrm{D}$ band also originate from the same double resonance phenomenon and correspond to a fully-dispersive $\mathrm{q}=2 \mathrm{k}$ while the $2450 \mathrm{~cm}^{-1}$ band correspond to non-dispersive $\mathrm{q}=0, \mathrm{k}$ for the electron and $\mathrm{q}$ for the phonon are measured from the K-point. From the corresponding phonon frequency and dispersion, the $2450 \mathrm{~cm}^{-1}$ band been modelled with an inter-valley process involving one iTO and one LA phonon and assigned as an overtone of LO phonon mode [38,39].

The relatively low intensity of the D-peak implies that the carbon atoms still maintain the honeycomb structure. The intensity of both the G- and 2D-peaks is greatly enhanced after the deposition of the Ag particles. The enhancement factors of the G- and 2D-bands of graphene after Ag deposition are $\sim 4.5$ and $\sim 5.5$ respectively. The low values could be attributed to the weak van der Waals interaction between Ag and graphene, which may change the structure of the graphene. The results here are similar to those obtained by Lee, Novoselov and Shin [40] who proposed that the Raman signal in Ag-deposited graphene is due to the first-order process and therefore that the interaction between Ag nanoparticles and graphene could lead to a change in the electronic structure.

The phase analysis of the samples was obtained by XRD measurement. Figure 2 shows the XRD patterns of the as-grown GF and GF/Ag samples. The peaks at $2 \theta$ values of $44.7^{\circ}$ and $51.9^{\circ}$ are assigned respectively to the (111) and (200) crystallographic planes of the fluid catalytic cracking (FCC) silver nanoparticles. Due to the small quantity and size broadening at higher angles of the silver nanoparticles, peaks such as the (200) and (220) at peak positions $51.9^{\circ}$ and $76.7^{\circ}$ are almost in the background. 


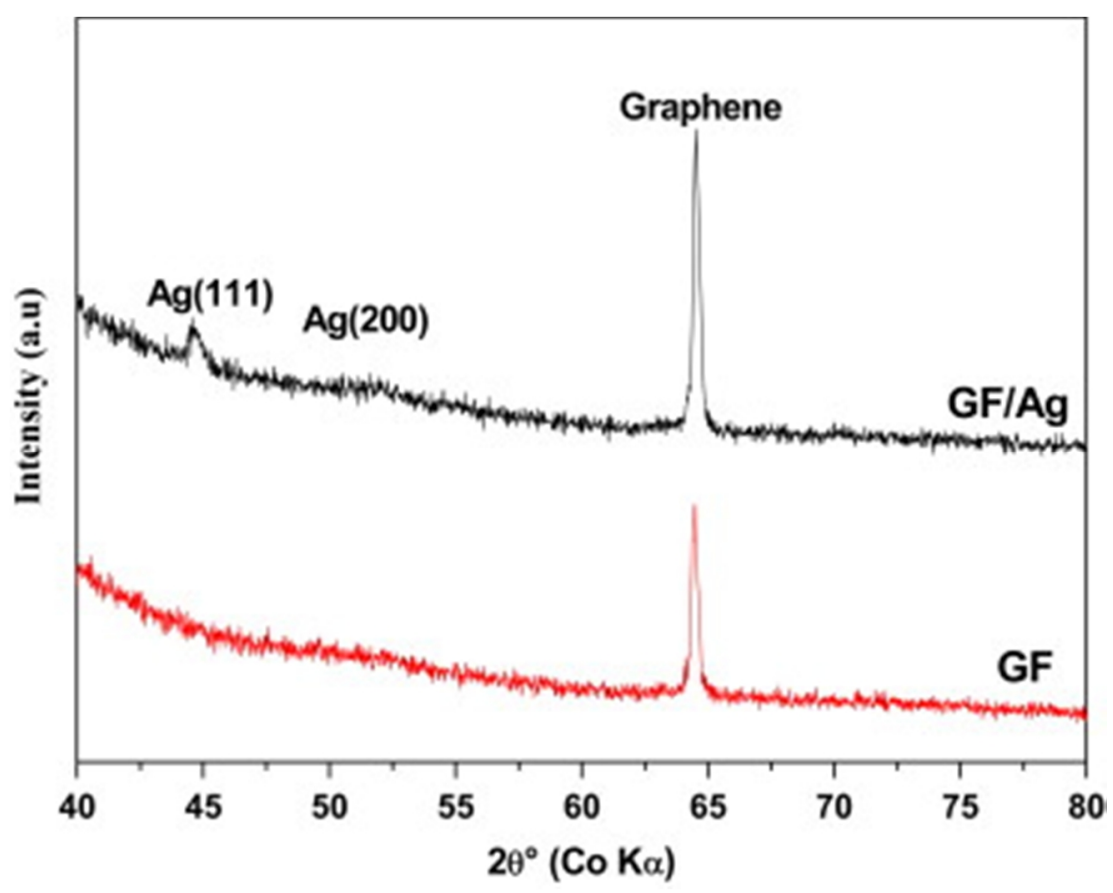

Figure 2. XRD patterns of samples: graphene foam (GF), and graphene foam with the silver nanoparticles (GF/Ag)
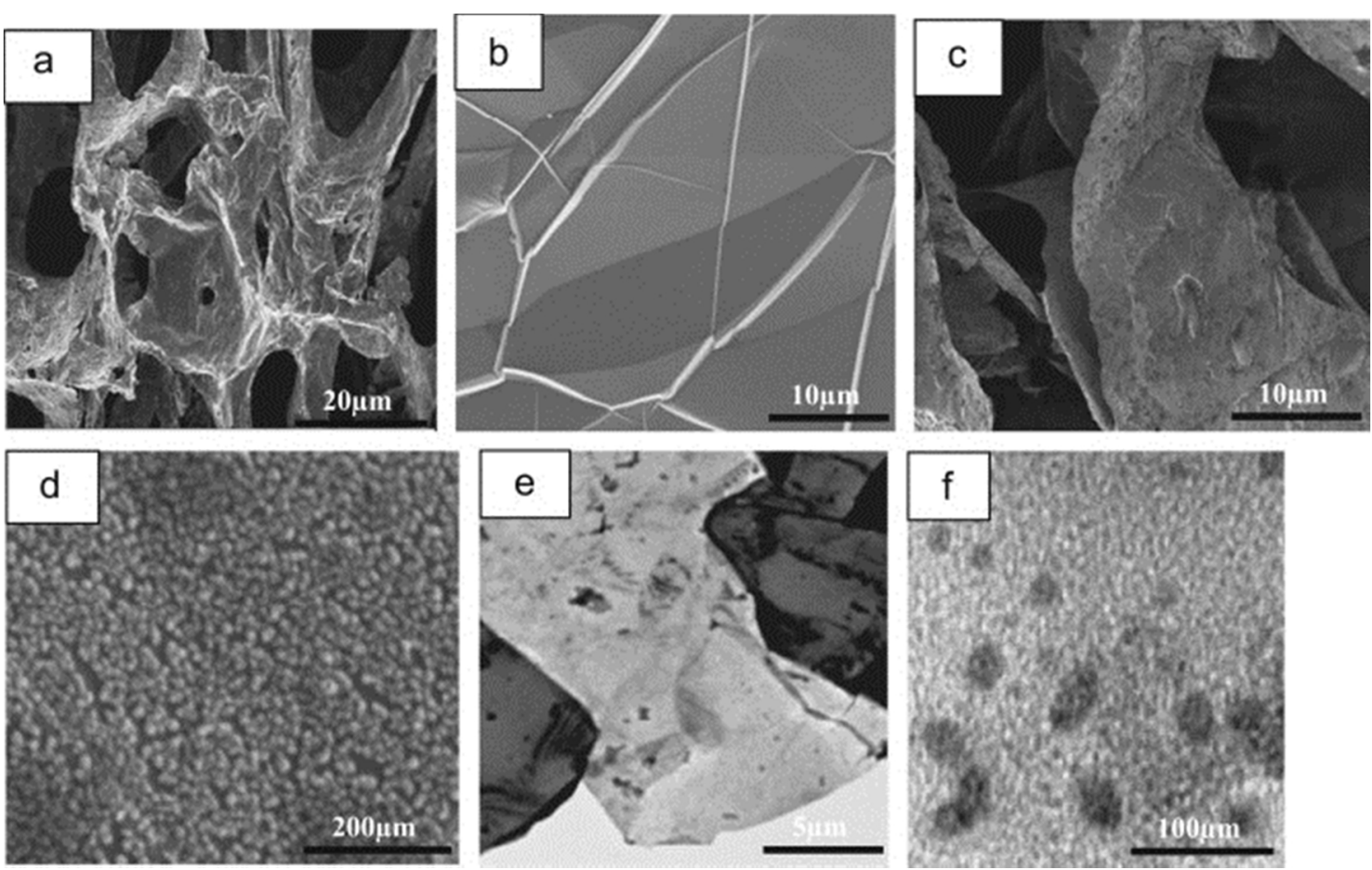

Figure 3. Scanning electron micrographs: $(\mathrm{a}, \mathrm{b})$ graphene foam $(\mathrm{GF})$; (c, d) graphene foam with the silver nanoparticles at different magnifications (GF/Ag); (e, f). Transmission electron micrographs: (GF) and (GF/Ag) 
The structure and morphologies of the graphene foam were also examined by FE-SEM and TEM. The scanning electron micrographs in Figure 3 (a) show that the GF takes the shape of the template and reveals a microporous nature. A high magnification of the GF is shown in Figure 3 (b) and reveals that the GF consists of wrinkles and ripples. The ripples are attributed to the different thermal expansion coefficients of nickel and graphene during the CVD synthesis, while the wrinkles can provide a high surface area and good mechanical properties [25,41]. Figure 3 (c, d) shows SEM images of the Ag nanoparticles decorated on the GF. Figure 3 (c) reveals uniform coverage of the GF by the Ag nanoparticles, while Figure 3 (d) shows that the silver nanoparticles are spherical and form agglomerates. TEM images of the GF and GF/Ag nanoparticles are shown in Figure 3 (e, f). Figure 3 (e) shows that the GF sheets overlap each other in the 3D network structure (the bright and dark regions respectively), while Figure 3 (f) shows that the GF is decorated by the Ag nanoparticles. The 3D and porous nature of the GF allowed the uniform distribution of the particles, as observed from both the SEM and TEM images.
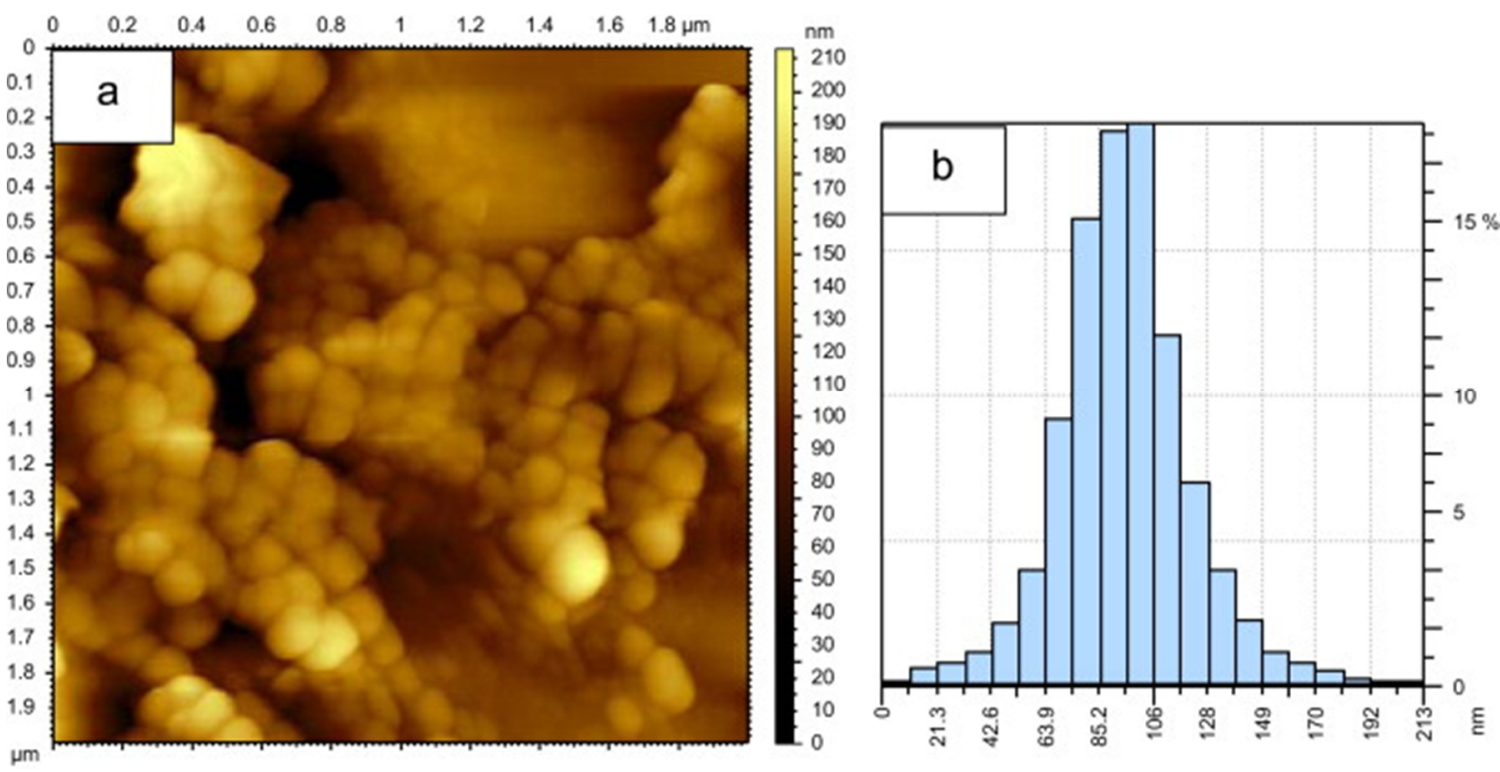

Figure 4. Atomic force micrographs: (a) GF/Ag showing the spherical nature of the particles and also revealing agglomeration; and (b) particle size distribution of the particles 
The atomic force micrographs in Figure 4 (a) show that the GF is decorated with $\mathrm{Ag}$ nanoparticles which are spherical and agglomerate on the surface. The average particle size was estimated to be $106 \mathrm{~nm}$. Figure 4 (b) shows the particle size distribution which reveals that more than $60 \%$ of the particles are within the range of $85 \mathrm{~nm}-106 \mathrm{~nm}$. The RMS surface roughness of the GF/Ag nanoparticles was $21.4 \mathrm{~nm}$.
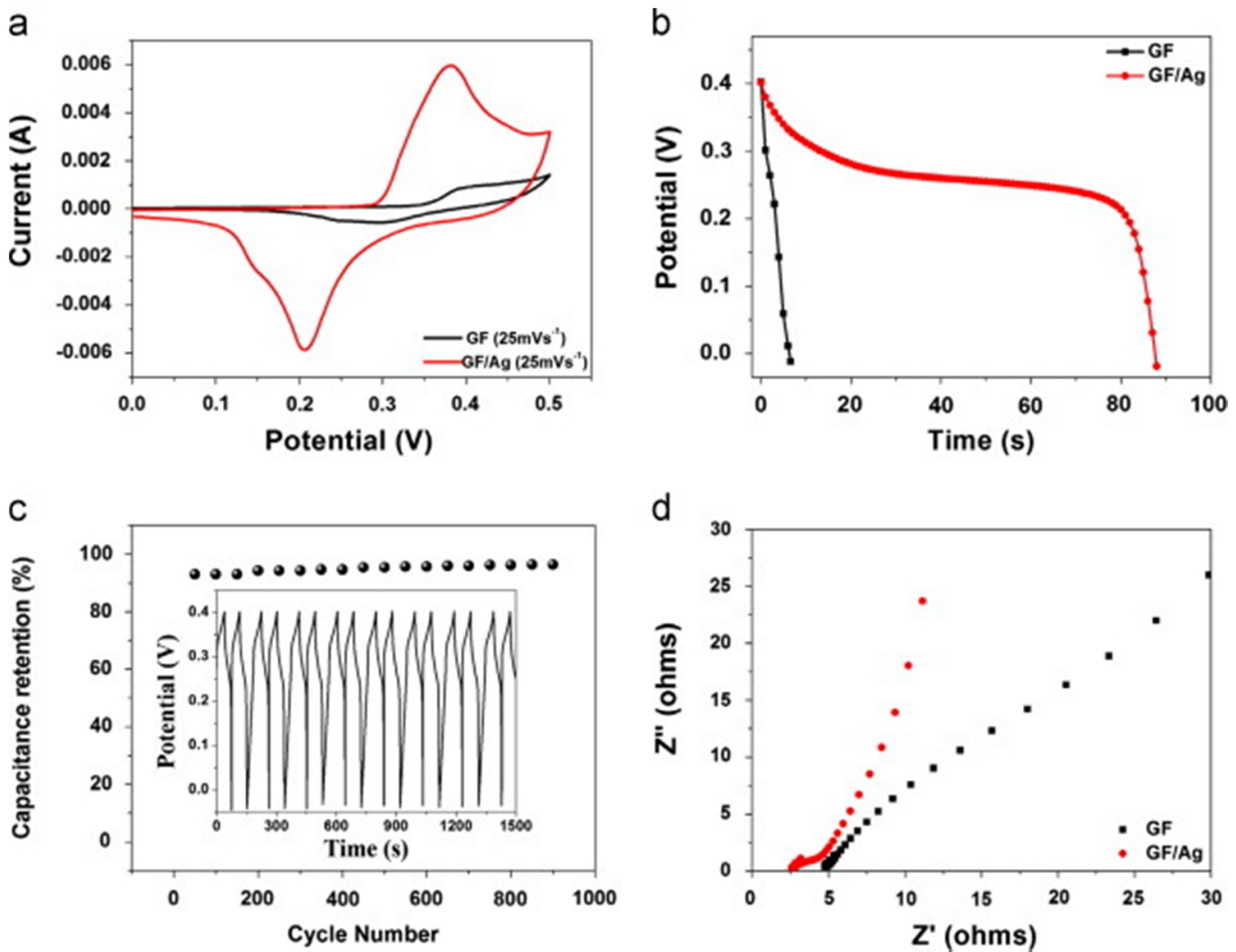

Figure 5. Electrochemical measurements of graphene foam (GF) and GF/Ag composites: (a) cyclic voltammetry of both GF and GF/Ag at $25 \mathrm{mVs}^{-1}$; (b) galvanostatic charge-discharge of both GF and GF/Ag measured at a current density of $0.5 \mathrm{Ag}^{-1}$; (c) capacitance retention of GF/Ag; (d) EIS plot of both GF and $\mathrm{GF} / \mathrm{Ag}$

Electrochemical studies were performed to investigate the effect of the Ag nanoparticles on the GF. The cyclic voltammetry (CV) and galvanostatic charge-discharge were measured in a $2 \mathrm{M} \mathrm{KOH}$ solution in the potential range from 0 to $0.5 \mathrm{~V}$, as shown in Figure 5. Figure 5 (a) shows the $\mathrm{CV}$ evolutions of the GF and GF/Ag at $25 \mathrm{mVs}^{-1}$. It can be seen that the GF 
showed insignificant current response compared with the GF/Ag. The weak redox couple observed on the GF is presumably due to the $\mathrm{Ni}^{2+} / \mathrm{Ni}^{3+}$ redox process arising from the nickel impurities during the etching process [42]. The well-defined redox process on the GF/Ag is related to the one-electron process of the Ag nanoparticles, the anodic current at the peak potential of $0.4 \mathrm{~V}$ (oxidation of $\mathrm{Ag}^{2+}$ to $\mathrm{Ag}^{3+}$ ) and the cathodic current at the peak potential of $0.2 \mathrm{~V}$ (reduction of $\mathrm{Ag}^{3+}$ back to $\mathrm{Ag}^{2+}$ ) [12]. The ratio of the anodic to cathodic peak current $\left(I_{\mathrm{pa}} / I_{\mathrm{pc}}\right)$ is approximately unity, clearly indicating that the redox reaction of silver nanoparticles on the GF is a reversible process.

Figure 5 (b) compares the galvanostatic charge-discharge curves of both the GF and GF/Ag nanoparticles at a constant current density of $0.5 \mathrm{Ag}^{-1}$. The non-linearity of the GF/Ag curves is due to the redox process of the silver nanoparticles on the GF (i.e. pseudocapacitance), corroborating the $\mathrm{CV}$ observed in Figure 5 (a)

The specific capacitance was calculated from the discharge curve using the following equation:

$C_{s}=\frac{I \Delta t}{\Delta V m}$

where $C_{s}$ is the specific capacitance, $m$ is the mass of working electrode, $I$ is the current (A), $\Delta V$ is the voltage applied and $\Delta t$ is the discharge time. The specific capacitance of the GF was estimated to be $\sim 10 \mathrm{Fg}^{-1}$, while that of the GF/Ag was about $110 \mathrm{Fg}^{-1}$. This increase in capacitance of the GF/Ag is due to the synergy arising from the high redox activity or Faradaic process (i.e. pseudocapacitance) of the silver nanoparticles, combined with the high conductivity and non-Faradaic process (i.e. electric double layer capacitance) of the GF. The 3D and porous nature of the GF also allows for a large and uniform deposition of the silver nanoparticles, thereby giving easy access to the electrolytes. Although the value of the 
specific capacitance is comparable with previous reported value [12], it high is compared to graphite and carbon materials $[45,46]$. It demonstrates the feasibility of having a graphene sample with a low defect density as compared with reduced graphene oxide which is normally regarded as being functionalized by the reducing agents, and also demonstrates the ability of e-beam evaporation to load nanoparticles for improved electrochemical and catalytic applications. Figure 5 (c) shows the continuous charge-discharge curve of the GF/Ag sample and the capacitance retention at a current density of $0.5 \mathrm{~A} / \mathrm{g}$. The electrode material is electrochemically stable after 1000 cycles and retains $93 \%$ of its initial capacitance.

EIS is an excellent tool for investigating the electrochemical characteristics of an electrode/electrolyte interface using a Nyquist plot, which is a representation of the real and imaginary parts of the impedance in the samples. The Nyquist plot of the electrode is shown in Figure 5 (d). It is worth stating that for ideal supercapacitors, the EIS (Nyquist) plot should be a line perpendicular to the imaginary axis. From the Nyquist plot, the slope at low frequencies can also be used to evaluate the capacitive performance [43]. However, a comparison of both samples shows that the Nyquist plot of GF/Ag is much closer to the ideal behaviour, thus indicating a better capacitive behaviour. This could be attributed to the threedimensionality of the graphene foam, which allows a uniform adsorption of silver nanoparticles, and to the high redox activity of the silver nanoparticles, coupled with the good conductivity of the GF.

The equivalent series resistance (ESR), including the resistance of aqueous electrolyte, the intrinsic resistance of the composite material and the contact resistance at the electrode interface, can be obtained from the X-intercept of the Nyquist plot [44]. As shown in Figure 5 (d), the ESR of the GF/Ag sample (2.6 $\Omega$ ) is much lower than that of the GF sample $(4.3 \Omega)$. 
These results are supported by electrical conductance measurements at room temperature for both GF and GF/Ag, for which the values of $\sim 7.6 \times 10^{-2} \mathrm{~S} / \mathrm{m}$ and $\sim 15 \times 10^{-2} \mathrm{~S} / \mathrm{m}$ were obtained respectively. It is clear from both results that GF/Ag exhibits half the ESR value and twice the conductance value with respect to those of GF. These results suggest that 3D GF can serve as an efficient support for metal nanoparticles for potential electrochemical applications, such as in the construction of electrochemical capacitors, electrocatalytic devices and electrochemical sensors.

\section{Conclusions}

In this work, GF was decorated with Ag nanoparticles using the electron beam evaporation technique and the effect of Ag on the electrochemical behaviour and specific capacitance of the GF/Ag composites was investigated. The interaction of the silver metal with the graphene foam was characterized using various microscopy and spectroscopy techniques. The morphologies reveal that the 3D structure of the GF provides support for adsorption and integration of the metal nanoparticles. Also, the enhanced electrochemical performance of the GF/Ag sample that was observed is due to the synergistic effect between the graphene and the silver nanoparticles, which could be attributed to the good electrical conductivity and uniform loading of the silver nanoparticles onto the surface of the GF. These results demonstrate that 3D GF could serve as an excellent platform for depositing metal nanoparticles for fundamental and applied electrochemical studies.

\section{Acknowledgements}

This work is based on research supported by the South African Research Chairs Initiative (SARCHi) of the Department of Science and Technology (DST) and the National Research Foundation (NRF). Any opinion, findings and conclusions or recommendations expressed in this work are those of authors and therefore the NRF and DST do not accept any liability with 
regard thereto. A. Bello. acknowledges the financial support from the University of Pretoria for his study. We thank Dr Patricia Forbes for supplying the nickel foams.

\section{References}

[1] A. Geim, K. Novoselov, Nature Mater. 6 (2007) 183-191.

[2] K.S. Novoselov, A.K. Geim, S.V Morozov, D. Jiang, M.I. Katsnelson, I.V. Grigorieva, S.V. Dubonos, A.A. Firsov, Nature. 438 Nature 438 (2005) 197-200.

[3] M.I. Katsnelson, Mater. Today 10 (2007) 20-27.

[4] K. Novoselov, A. Geim, S. Morozov, Science 306 (2004) 666-669.

[5] M.D. Stoller, S. Park, Y. Zhu, J. An, R.S. Ruoff, Nano Lett. 8 (2008) 3498-502.

[6] Y. Wang, Z. Shi, Y. Huang, Y. Ma, C. Wang, M. Chen, Y.S. Chen, J. Phys.Chem.C. 113 (2009) 13103-13107.

[7] Z-S. Wu, W. Ren, D-W. Wang, F. Li, B. Liu, H-M. Cheng, ACS Nano 4 (2010) 58355842.

[8] Y. Zhu, S. Murali, W. Cai, X. Li, Adv. Mater. 22 (2010) 3906-3924.

[9] Y. Sun, Q. Wu, G. Shi, Energy Environ. Sci. 4 (2011) 1113-1132.

[10] X. Huang, X. Qi, F. Boey, H. Zhang, Chem. Soc. Rev. 41 (2012) 666-686.

[11] S. Park, R.S. Ruoff, Nat. Nanotechnol. 4 (2009) 217-224.

[12] K-S. Kim, I-J. Kim, S-J. Park, Synthetic Metals 160 (2010) 2355-2360.

[13] X. Sun, M. Xie, G. Wang, H. Sun, A.S. Cavanagh, J.J. Travis, S.M. George, L. Jie, J. Electrochem. Soc. 159 (2012) A364-A369.

[14] X. Dong, Y. Cao, J. Wang, M.B. Chan-Park, L. Wang, W. Huang, W.H. Peng, RSC Adv. 2 (2012) 4364-4369.

[15] X. Li, B.K. Tay, J. Li, D. Tan, C.W. Tan, K. Liang, Nanoscale Res. Lett. 7 (2012) 205.

[16] S. Liu, J. Tian, L. Wang, X. Sun, Carbon. 49 (2011) 3158-3164.

[17] Y-K. Yang, C-E. He, W-J. He, L-J. Yu, R-G. Peng, X-L. Xie, W. Xian-Bao, M. YiuWing, J Nanoparticle Res. 13 (2011) 5571-5581.

[18] J. Tian, S. Liu, Y. Zhang, H. Li, L. Wang, Y. Luo, A.M. Asiri, A.O. Al-Youbi, X. Sun, Inorganic Chem. 51 (2012) 4742-4746. 
[19] G.F. Ndlovu, W.D. Roos, Z.M. Wang, J.K. Asante, M.G. Mashapa, C.J. Jafta, B.W. Mwakikunga, K.T. Hillie, Nanoscale Res. Lett. 7 (2012) 173.

[20] Y.H. Kahng, S. Lee, W. Park, G. Jo, M. Choe, J-H. Lee, Y. Hyunung, L. Takhee, L. Kwanghee, Nanotechnol. 23 (2012) 075702.

[21] H. Wang, H.S. Casalongue, Y. Liang, H. Dai, J. Am. Chem. Soc. 132 (2010) 74727477.

[22] Z. Chen, W. Ren, L. Gao, B. Liu, S. Pei, H. Cheng, Nature Mater. 10 (2011) 424-428.

[23] T. Maiyalagan, X. Dong, P. Chen, X. Wang, J. Mater. Chem. 22 (2012) 5286-5290.

[24] H. Ji, L. Zhang, M. Pettes, H. Li, S. Chen, Nano Lett. 12 (2012) 2446-2451.

[25] X. Cao, Y. Shi, W. Shi, G. Lu, X. Huang, Q. Yan, Q. Zhang, H. Zhang, Small 7 (2011) 3163-3168.

[26] J-S. Lee, H-J. Ahn, J-C. Yoon, J-H. Jang, Phys. Chem. Chem. Phys. Phys, Phys2011) 7943.

[27] T. Lu, Y. Zhang, H. Li, L. Pan, Y. Li, Z. Sun, Electrochim. Acta. 55 (2010) 41704173.

[28] X-C. Dong, H. Xu, X-W. Wang, Y-X. Huang, M.B. Chan-Park, H. Zhang, L-H Wang, W Huang, P. Chen, ACS Nano. 6 (2012) 3206-3213.

[29] C.M. Welch, C.E. Banks, A O. Simm, R.G. Compton, Anal. Bioanal. Chem. 382 (2005) 12-21.

[30] X. Dai, G.G. Wildgoose, R.G. Compton, Analyst 131 (2006) 1241-1247.

[31] M. Tominaga, T. Shimazoe, M. Nagashima, H. Kusuda, A. Kubo, Y. Kuwahara, T. Isao J. Electroanal. Chem. 590 (2006) 37-46.

[32] F.W. Campbell, S.R. Belding, R. Baron, L. Xiao, R.G. Compton, J. Phys. Chem. C. 113 (2009) 14852-14857.

[33] M. Chatenet, M. Aurousseau, R. Durand, F. Andolfatto, J. Applied Electrom. 263 (2002) 1131-1140.

[34] A. Bello, K. Makgopa, M. Fabiane, D. Dodoo-Ahrin, K.I. Ozoemena, N. Manyala, J. Mat. Sci. 48 (2013) 6707-6712

[35] A.C. Ferrari, J.C. Meyer, V. Scardaci, C. Casiraghi, M. Lazzeri, F. Mauri, S. Piscanec, D. Jiang, K.S. Novoselov, S. Roth, A.K. Geim, Phys. Rev. Lett. 97 (2006) 1-4.

[36] M.S. Dresselhaus, A. Jorio, M. Hofmann, G. Dresselhaus, R. Saito, Nano Lett. 10 (2010) 751-758. 
[37] T. Shimada, T. Sugai, C. Fantini, M. Souza, L.G. Cancado, A. Jorio,M.A. Pimenta, R. Saito, A. Grüneis, G. Dresselhaus, M.S. Dresselhaus, Y. Ohno, T. Mizutani, H. Shinohara Carbon 43 (2005) 1049-1054

[38] D. L. Mafra, G. Samsonidze, L. M. Malard, D. C. Elias, J. C. Brant, F. Plentz, E. S. Alves, and M. A. Pimenta, Physical Review B 76 (2007) 233407

[39] L.M. Malard, M.A. Pimenta, G. Dresselhaus, M.S. Dresselhaus, Physics Reports 473 (2009) 51-87

[40] J. Lee, K. Novoselov, H. Shin, ACS Nano. 5 (2010) 608-612.

[41] S.J. Chae, F. Güneş, K.K. Kim, E.S. Kim, G.H. Han, S.M. Kim, H.J. Shin, S. Yoon, J. Choi, M.H. Park, C.W. Yang, D. Pribat, Y.H. Lee, Adv. Mater. 21 (2009) 2328-2333.

[42] L-R. Zhang, J. Zhao, M. Li, H-T. Ni, J. Zhang, X-M. Feng, Y-W. Ma, Q-L. Fan, X-Z. Wang, Z. Hu, W. Huang, New J.Chem. 36 (2012) 1108.

[43] E. Frackowiak, F. Béguin, Carbon. 39 (2001) 937-950.

[44] J. Zhang, L-B. Kong, J-J. Cai, Y-C. Luo, L. Kang, J. Solid State Electrochem. 14 (2010) 2065-2075.

[45] T. Cassagneau, J. H. Fendler, J. Phys. Chem. B (1999) 1789 - 1793.

[46] L. Jiang, M. Vangari, T. Pryor, Z. Xiao, N.S. Korivi, Microelectronic Engineering (2013) 52-57 